\section{SUCCESSFUL GENERATION OF TUMOR-INFILTRATING LYMPHOCYTE (TIL) PRODUCT FROM RENAL CELL CARCINOMA (RCC) TUMORS FOR ADOPTIVE CELL THERAPY}

'Brian Halbert, 'David Einstein, 'David McDermott, 'Emanuelle Andrianopoulos, ${ }^{1}$ Mamta Gupta, ${ }^{1}$ Virginia Seery, ${ }^{2}$ Kenneth Onimus, ${ }^{2}$ Courtney Herman, ${ }^{2}$ Adrian Wells, ${ }^{2}$ Shwetha Lakshmipathi, ${ }^{2}$ Arvind Natarajan, ${ }^{2}$ Anand Veerapathran, ${ }^{1}$ Rupal Bhatt, 'Brian Halbert*. 'BIDMC, Boston, MA, USA; ${ }^{2}$ lovance Biotherapeutics, San Carlos, CA, USA

Background Patients with RCC may achieve remission with immune-checkpoint inhibitors (ICI); however, most patients will progress. Adoptive cell therapy with autologous TIL allows for expansion of T-cells from tumor tissue leading to a polyclonal T-cell product with a diverse $\mathrm{T}$-cell receptor repertoire capable of recognizing an array of tumor antigens. TIL therapy with centrally manufactured lifileucel demonstrated a $36 \%$ overall response rate in patients with ICI-refractory melanoma. ${ }^{1}$ We present our preclinical experience of TIL production in RCC.

Methods This study was approved by the DF/HCC Institutional Review Board. Fresh tumor samples $(\geq 1.5-\mathrm{cm})$ were harvested from consented patients undergoing resection for RCC. TIL were manufactured using pre-rapid expansion (1/ 10th scale) and rapid expansion (1/100th scale) protocol for 22 days. Characterization (total viable cells [TVC],\% viability, identity, and potency) was performed on the final TIL product. TIL purity, differentiation, memory, activation, and exhaustion status were characterized using multi-color flow cytometry.

Results Baseline characteristics of 11 recruited patients are shown in table 1. Clear cell was the most common histology (73\%). Two patients had previously treated metastatic disease with samples harvested from the lung and adrenal gland; one patient had prior cryoablation with adjacent local recurrence. The remainder were treatment-naïve primary nephrectomy samples. Eight products (73\%) showed acceptable TIL product attributes (table 2). Median (range) TVC, viability, and identity $(\mathrm{CD} 45+\mathrm{CD} 3+\%)$ for the final TIL product were $74 \times 109$ $(18 \times 109-133 \times 109), 95 \%(86 \%-97 \%)$, and 98\% (94\%-99\%), respectively. Median (range) percentage of CD4+ cells was $69 \%(21 \%-97 \%)$ and CD8 + cells was 27\% (2\%-72\%). The non-T-cell population, including $\mathrm{B}$ cells, monocytes, and NK cells, was $<7 \%$. The final TIL product was functional, and responded to polyclonal bead stimulation; the median (range) IFNg and granzyme B were $8834(3319-12,957) \mathrm{pg} / \mathrm{mL}$ and $34,329(15,565-65,521) \mathrm{pg} / \mathrm{mL}$, respectively. Acceptable TIL product was generated from both metastatic lesions and primary tumor samples. Of the 3 tumor samples that did not demonstrate acceptable TIL product attributes, one (ID 1) was from a patient treated with a CXCR4 inhibitor $<1$ month before resection, another (ID 4) was from a patient previously treated with ocrelizumab (CD20-directed cytolytic antibody) for multiple sclerosis, and the final (ID 8) was harvested from the cryoablated tumor nodule rather than the adjacent recurrent tumor.

Conclusions These feasibility data suggest that TIL can be successfully expanded ex vivo from RCC samples (including pretreated and metastatic tumors) and support potential clinical investigation of TIL in patients with RCC.
Abstract 176 Table 1 Baseline demographics and tumor characteristics

\begin{tabular}{lc}
\hline & $\mathrm{N}=11$ \\
\hline $\begin{array}{l}\text { Age, years, median (IQR) } \\
\text { Sex, } \mathrm{n}(\%)\end{array}$ & $59(52-68)$ \\
$\quad$ Male & $10(91)$ \\
Race, $\mathrm{n}(\%)$ & \\
$\quad$ White & $9(82)$ \\
Histology, $\mathrm{n}(\%)$ & \\
$\quad$ Clear cell & $8(73)$ \\
Papillary & $2(18)$ \\
$\quad$ Chromophobe & $1(9)$ \\
Tumor site, $\mathrm{n}(\%)$ & \\
$\quad$ Kidney & $9(82)$ \\
Adrenal & $1(9)$ \\
Lung & $1(9)$
\end{tabular}

Abstract 176 Table 2 Summary of product attributes. 1, No CD3 + subset. 2, Product not available to test

\begin{tabular}{c|llccccccc}
$\begin{array}{c}\text { Tumor } \\
\text { ID }\end{array}$ & Histology & Tumor & $\begin{array}{c}\text { Acceptance } \\
\text { Criteria }\end{array}$ & $\begin{array}{c}\text { TVC } \\
\left(\times 10^{9}\right)\end{array}$ & $\begin{array}{c}\text { Viability } \\
(\%)\end{array}$ & $\begin{array}{c}\mathrm{CD}^{2} 5^{+} \mathrm{CD}^{+} \\
(\%)\end{array}$ & $\begin{array}{c}\mathrm{CD} 4^{+} \\
(\%)\end{array}$ & $\begin{array}{c}\mathrm{CD}^{+} \\
(\%)\end{array}$ & $\begin{array}{c}\mathrm{CD} 4^{+1} \\
\mathrm{CD8}^{+}\end{array}$ \\
\hline 1 & Clear cell & Lung & Not met & 1 & 64 & 0 & & -1 & \\
2 & Clear cell & Primary & Met & 77 & 97 & 99 & 48 & 34 & 1 \\
3 & Clear cell & Primary & Met & 96 & 95 & 96 & 21 & 72 & 0 \\
4 & Clear cell & Primary & Not met & 2 & 67 & 0 & & -1 & \\
5 & Clear cell & Primary & Met & 71 & 91 & 99 & 77 & 18 & 4 \\
6 & Papillary & Primary & Met & 63 & 95 & 98 & 58 & 29 & 2 \\
7 & Papillary & Primary & Met & 81 & 94 & 99 & 97 & 2 & 49 \\
8 & Clear cell & Primary & Not met & 1 & 92 & 75 & & -2 & \\
9 & Chromophobe & Primary & Met & 27 & 91 & 94 & 72 & 25 & 3 \\
10 & Clear cell & Primary & Met & 113 & 95 & 99 & 65 & 29 & 2 \\
11 & Clear cell & Adrenal & Met & 18 & 86 & 94 & 80 & 18 & 4
\end{tabular}

\section{REFERENCES}

1. Sarnaik AA, Hamid O, Khushalani NI, Lewis KD, Medina T, Kluger HM, Thomas SS, Domingo-Musibay E, Pavlick AC, Whitman ED, Martin-Algarra S, Corrie $P$, Curti BD, Oláh J, Lutzky J, Weber JS, Larkin JMG, Shi W, Takamura T, Jagasia M, Qin $\mathrm{H}$, Wu X, Chartier C, Graf Finckenstein F, Fardis M, Kirkwood JM, Chesney JA. Lifileucel, a Tumor-Infiltrating Lymphocyte Therapy, in Metastatic Melanoma. J Clin Oncol 2021 May 12:JCO2100612. doi: 10.1200/JCO.21.00612. Epub ahead of print. PMID: 33979178.

Ethics Approval This study was approved by the DF/HCC Institutional Review Board protocol \# 06-105.

http://dx.doi.org/10.1136/jitc-2021-SITC2021.176 